\title{
NUMERICAL SIMULATIONS OF THE EFFECT OF TURBULENCE IN THE THERMAL-RADIATION FLOW FIELD
}

\author{
O. M. Oyewola ${ }^{a, b,{ }^{*}}$ O. S. Ismail ${ }^{b}$ and J. O. Bosomo ${ }^{b}$ \\ ${ }^{a}$ School of Mechanical Engineering, Fiji National University, Suva, Fiji. \\ ${ }^{b}$ Department of Mechanical Engineering, University of Ibadan, Ibadan, Nigeria.
}

\begin{abstract}
This paper investigates possible inherent modifications of the radiative heat source term due to the influence of turbulence in the thermal radiation field of a gas turbine combustor flame. Adapting a flame temperature of $2000[\mathrm{~K}]$, COMSOL Multiphysics software was utilized to numerically simulate the process, assuming a gray gas participating medium with absorption coefficient of $0.03\left[\mathrm{~m}^{-1}\right]$. The analysis of the results for five (5) different radial cut sections of the simulated combustor chamber shows that turbulence-radiation interactions cause radiative heat losses from the flame, with the divergence of the radiative heat flux having a deviation factor of 3.48 , and a $1.43 \%$ reduction in the local flame temperature, as observed at a critical radial section of the combustor chamber.

Keywords: Radiative heat source term, gas turbine combustor, flame temperature, turbulence-radiation interactions
\end{abstract}

\section{INTRODUCTION}

Radiation has a fourth-power dependence on temperature, making it a dominant source of heat transfer for very high temperature processes. Of enormous engineering and scientific importance are its numerous applications in almost all fields associated with high temperature, energy conversion processes, and particularly in the design of various thermal devices; the combustion process in gas turbine combustors is a common application. Most practical combustion devices, however, operate in turbulent flow conditions. The need to accurately model the combustion process is paramount, and cannot be overemphasized, as increasing regulations on emissions from combustion devices, higher efficiency considerations, and hence, energy conservations are global issues (Ian, 1997; Masataka et al 2019).

It should be noted that simulation has played significant roles in advancing progress in engineering and science. Consequently, it will continue to have great impact on all areas of engineering and scientific discovery because of its versatility (Babuska and Oden, 2004). In numerical simulations of high energy dependent processes, accurate treatment of radiation heat transfer is essential. The influence of turbulent fluctuations in the thermal radiation field is obvious from the fluctuations in the radiation intensity, which is a function of the scalar temperature field. Therefore, the fluctuations of the scalar field as a result of the turbulence flow field, causes a corresponding fluctuation in the thermal radiation field, resulting to modifications of the energy sources associated with the process; a phenomenon termed Turbulence-Radiation Interactions (TRI) (Li and Modest, 2003; Tessé, et al., 2004; Modest, 2003). However, these interactions had until recently been ignored by virtually all investigations due to the complexities associated with the non-linear couplings between radiation and turbulence models. While the investigations on TRI was originated in the 1960's, the first numerical study was achieved in 1978 (Germano, 1978), TRI has received attention in recent years (Li and Modest, 2002; Coelho, 2007). Reviews showed that accounting for TRI can increase radiative losses from a flame by factors more than two (2) (Faeth et al, 1989; Li and Modest, 2002). Many experimental and numerical results have also shown that neglecting TRI can result in underestimation of heat loss by factors up to three (3) (Townsend, 1958; Song, and Viskanta, 1987). Dos Santos et al, (2009) simulated the effect of TRI for a flame previously simulated by (Barve et $a l, 2006)$. They utilized a one-dimensional temperature profile predicted from the temperature of the simulated flame at just one cut section of the cylindrical chamber. They discovered that TRI contributed significantly to radiative flux especially at higher temperatures.

Despite the previous work, there is still a lot of unresolved issues especially the effect of turbulence flow field on TRI. Therefore, the present work which is adapting to the gas turbine combustor system focuses on the numerical study of the effect of turbulence in the thermalradiation flow field taking into consideration the effect of TRI. The study will improve our understanding of the interaction in the flow field. The numerical simulation is performed using COMSOL Multiphysics software, incorporating advantages of flexibility in analysis for the comparison of five different cut sections of the combustor chamber, with flame temperature adapted from previous investigations on the adiabatic flame temperature in gas turbine combustors (Barths et al, 2000).

\section{THEORETICAL BACKGROUND}

Consider a 2-D cylindrical combustor chamber of height, $72 \mathrm{~m}$ and radius $20 \mathrm{~m}$ as shown in Fig. 1. Two identical nozzles of height $5 \mathrm{~m}$ are place symmetrically in the domain. With the aim, to investigate the influence of turbulent fluctuations primary for the estimation of the divergence of the radiative flux $\left(\nabla \cdot \mathbf{q}_{R}\right)$, without necessarily considering the spectral effects of the radiation field and the chemical kinetics associated with the

\footnotetext{
${ }^{*}$ Corresponding author. Email: olanrewaju.oyewola@fnu.ac.fj
} 
combustion process. The radiative intensity of the simulated flame is shown in Fig. 2.

To take into account the effect of the interaction between radiation and turbulence, a coupled model of both physics were treated. As a prerequisite for the simulation of the coupled process, a workable instance (converged solutions) of each of the two physics models is required.

The simplified form of the radiative transfer equation (RTE) in one dimension, eliminating complications concerned with the spectral effects, is given as:

$\frac{\mathrm{dI}}{\mathrm{dx}}=-\mathrm{kI}+\mathrm{kI}_{\mathrm{b}}$

$I=$ radiation intensity; $I_{b},=$ blackbody radiation intensity; $K=$ absorption coefficient

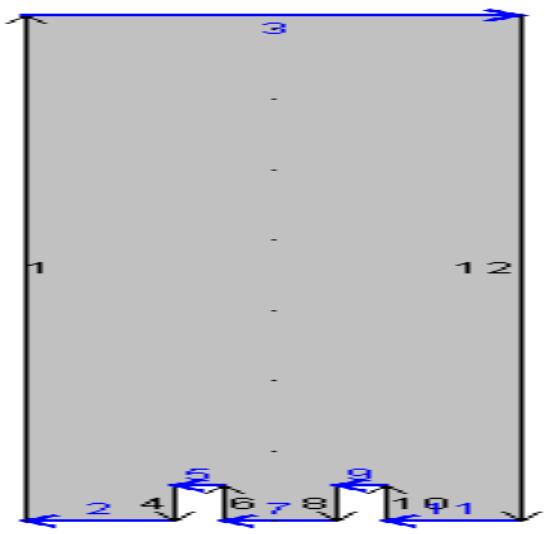

Fig. 1 The 2-D cylindrical domain

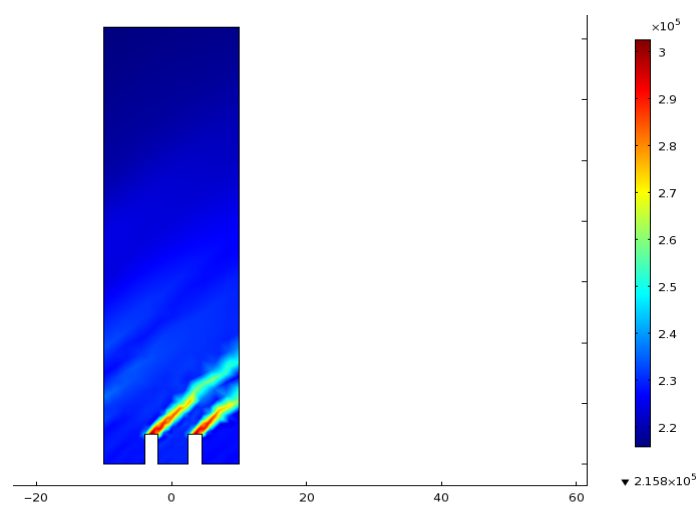

Fig. 2 Surface plot of the radiative intensity of the flame

The basic assumption of a gray gas participating medium, with absorption coefficient of $0.03\left[\mathrm{~m}^{-1}\right]$ (Dos Santos et al, 2009) is utilized. The turbulence model is based on the Reynold's Averaged Navier-Stokes equation (Riyadh et al 2019):

$\rho \frac{\partial u}{\partial t}+\rho(u \cdot \nabla) u+\nabla \cdot \overline{\left(\rho u^{\prime} \otimes u^{\prime}\right)}=-\nabla p+\nabla \cdot \mu\left(\nabla u+(\nabla u)^{T}+F\right.$

Where, $u \& u^{\prime}=$ averaged and fluctuating velocity respectively; $\otimes=$ outer vector product. The term $\nabla . \overline{\left(\rho u^{\prime} \otimes u^{\prime}\right)}$, is the interaction between the fluctuating velocities called Reynolds stress tensor.

A flame temperature of $2000[\mathrm{~K}]$ was utilized for the study, based on investigations on the adiabatic flame temperature in the gas turbine combustors (Barths et al, 2000). This is the temperature of the hot gases after the oxidation process in the combustor.

The effect of turbulence in the thermal radiation field is noticeable from the radiative heat flux $\left(\nabla \times \boldsymbol{q}_{R}\right)$, in simplified gray form is given as:

$$
\nabla \times \mathrm{q}_{\mathrm{R}}=\mathrm{k}\left[4 \pi \mathrm{I}_{\mathrm{b}}-\int_{4 \pi} \mathrm{Id} \Omega\right]
$$

This term is required as a source term in the energy equation, accounting for the effect of radiation field for investigation of the total energy characteristics of the system.

\subsection{Numerical modeling}

The simulation study was performed using the COMSOL Multiphysics Version 4.0a software. The Radiative Transfer Equation (RTE) was solved using the built-in Discrete Ordinate Method (DOM) which involved independent discretization of spatial and angular domains. The RTE equation is replaced by a discrete set of equations for a finite number of directions, $\Omega_{m}$, and each integral is replaced by a quadrature.

$\Omega_{m} \frac{\mathrm{dI}}{\mathrm{dx}}=-k \mathrm{I}+\mathrm{kI}_{b}$

Equation (4) is separated into two equations, one for positive direction and the other for negative direction, while the integral in Eq. (3) is replaced by a quadrature:

$\Omega \frac{\mathrm{dI}^{+}}{\mathrm{dx}}=-k \mathrm{I}^{+}+\mathrm{kI}_{b}$

$-\Omega \frac{\mathrm{dI}^{-}}{\mathrm{dx}}=-k \mathrm{I}^{-}+\mathrm{kI}_{b}$

$\nabla \times \mathrm{q}_{\mathrm{R}}=4 \pi \mathrm{kI} \mathrm{b}-2 \pi \mathrm{k} \sum_{k=1}^{\mathrm{M}} w_{k}\left[\mathrm{I}^{+}-\mathrm{I}^{-}\right]$

Where, $w_{k}$ are the ordinate weights.

This angular approximation is used to transform the original integraldifferential equation into a set of coupled differential equations. The discrete ordinate equations may then be discretized spatially after angular discretization has been performed using the finite volume method.

The physics used for the study are the heat transfer in participating media (htpm) and the turbulence flow, k-e (spf) model. It should be noted that the heat transfer in participating media interface provides the possibility to model radiative transfer inside a participating medium in conjunction with heat interaction in solids and fluids. The interface solves for radiative intensity and temperature fields. The turbulence flow interface provides the equations, boundary conditions, and volume forces for modeling turbulent flow, solving for the mean velocity field, the turbulent kinetic energy $\mathrm{k}$ and the rate of dissipation of turbulent kinetic energy, $\varepsilon$. Presented in Table 1 is the simulation model input.

\subsection{Boundary conditions}

Case 1: The domain is confined to the radiation field only, thereby neglecting the effects of the flow field on the radiation field

Case 2: The turbulence field is introduced into the domain with the aim of investigating its effect on the radiation field

Case 1 Boundary conditions:

Walls 1-4, 6-8, 10-12 are assumed to be black walls

The radiation intensities,

$I^{-}(x=-10 \mathrm{~m})=I_{b}(x=-10 \mathrm{~m})$

$I^{+}(x=10 \mathrm{~m})=I_{b}(x=10 \mathrm{~m})$,

Case 2 Boundary conditions:

Walls $5 \& 9$ has inlet boundary condition with inlet velocity of $10 \mathrm{~m} / \mathrm{s}$ 
Table 1 Simulation model input.

\begin{tabular}{|l|c|c|l|}
\hline \multicolumn{1}{|c|}{ Name } & Symbol & Unit & \multicolumn{1}{c|}{ Value } \\
\hline $\begin{array}{l}\text { Absorption } \\
\text { coefficient }\end{array}$ & $K$ & $\mathrm{~m}^{-1}$ & 0.03 (Dos Santos et al, 2008) \\
\hline $\begin{array}{l}\text { Scattering } \\
\text { coefficient }\end{array}$ & $\sigma_{s}$ & $\mathrm{~m}^{-1}$ & 0 \\
\hline $\begin{array}{l}\text { Thermal } \\
\text { conductivity }\end{array}$ & $K$ & $\mathrm{~W} /(\mathrm{m} . \mathrm{K})$ & 0.0257 \\
\hline $\begin{array}{l}\text { Heat } \\
\text { capacity }\end{array}$ & $C p$ & $\mathrm{KJ} / \mathrm{kgK}$ & 1.005 \\
\hline $\begin{array}{l}\text { Dynamic } \\
\text { viscosity }\end{array}$ & $\mu$ & $\mathrm{Pa} . \mathrm{s}$ & $1.79 \times 10^{3}$ \\
\hline Density & $\rho$ & $\mathrm{kg} / \mathrm{m}^{3}$ & 1.23 \\
\hline $\begin{array}{l}\text { Wall } \\
\text { Temperature }\end{array}$ & $T_{w}$ & $\mathrm{~K}$ & 293.15 \\
\hline $\begin{array}{l}\text { Radiation } \\
\text { intensity }\end{array}$ & $I$ & $\mathrm{~W}$ & Default (Inbuilt) \\
\hline $\begin{array}{l}\text { Surface } \\
\text { radiosity }\end{array}$ & $J$ & $\mathrm{~W} / \mathrm{m}^{2}$ & 0 \\
\hline $\begin{array}{l}\text { Inlet } \\
\text { velocity }\end{array}$ & $U_{o}$ & $\mathrm{~m} / \mathrm{s}$ & 10 \\
\hline $\begin{array}{l}\text { Flame } \\
\text { temperature }\end{array}$ & $T$ & $\mathrm{~K}$ & 2000 ( Barths et al, 2000) \\
\hline
\end{tabular}

\subsection{Discretization and Meshing of the domain}

A refine mesh of the entire geometry was performed using the regular refinement method. The mesh description is shown in Table 2.

Table 2 Mesh description.

\begin{tabular}{|l|c|c|c|}
\hline \multicolumn{1}{|c|}{ Element type } & Triangular & Edge & Vertex \\
\hline Number of elements & 996 & N/A & N/A \\
\hline Minimum element quality & 0.8208 & N/A & N/A \\
\hline Average element quality & 0.957 & N/A & N/A \\
\hline Element area ratio & 0.07485 & N/A & N/A \\
\hline Mesh area & $1420 \mathrm{~m}^{2}$ & N/A & N/A \\
\hline Element length ratio & N/A & 0.3043 & N/A \\
\hline Mesh edge length & N/A & $204 \mathrm{~m}^{2}$ & N/A \\
\hline Vertex elements & N/A & N/A & 12 \\
\hline
\end{tabular}

\section{RESULTS AND DISCUSSION}

The analysis of the temperature profiles and divergences of the radiative heat flux at five (5) different radial sections of the cylindrical combustor chamber with the purpose, to make comparison, and to study the behavior of the system along the transverse sections with and without the influence of TRI are presented in Fig.(3) Fig.(7).

It is evidenced from the plots of the temperature profiles and the divergence of the radiative flux for the different cut sections presented that the results follow a similar trend. It should be noted that with the influence of turbulence, the divergence of the radiative heat flux increases considerably, with corresponding drop in the peak temperature levels of the local flame temperature. This may be as a result of greater emissions and increased heat loss from the heat source. However, these emissions may adversely affect the performance and efficiency of the combustor. For instance, it could result in possible damping of the exergy of the system, with some of the energy needed for effective work distributed to the surfaces of the combustor and thereby result in thermal loading of the system. This may lead directly to the deterioration of the material of the combustor which eventually may result in premature failure of the combustor.

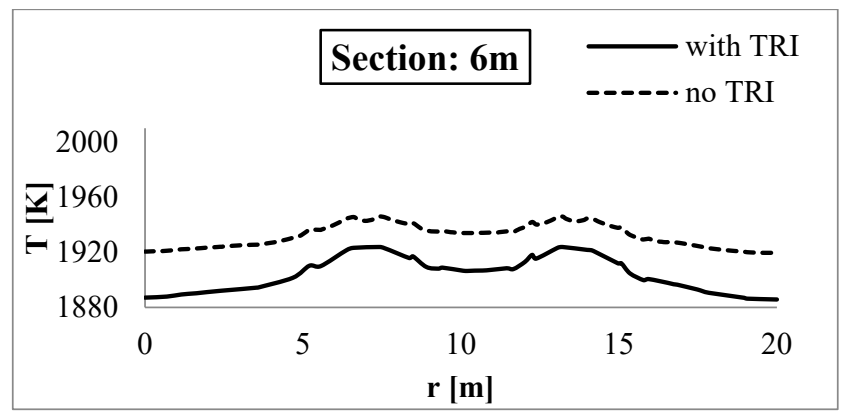

(a)

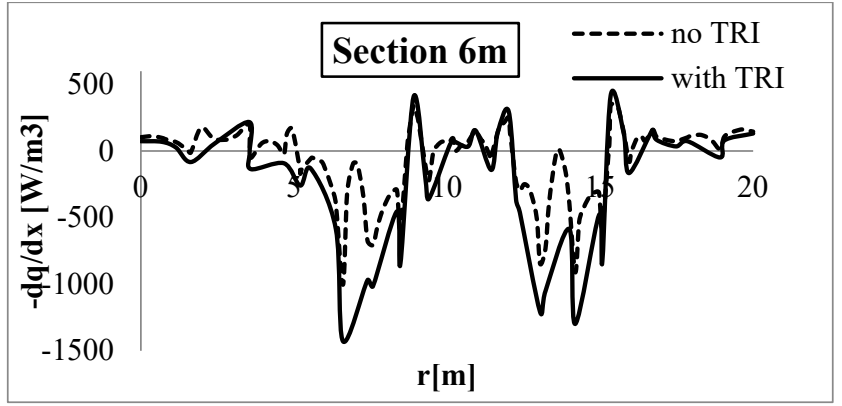

(b)

Fig. 3 (a) Temperature profile at section $6 m$, (b) Divergence of the radiative flux at section $6 \mathrm{~m}$.

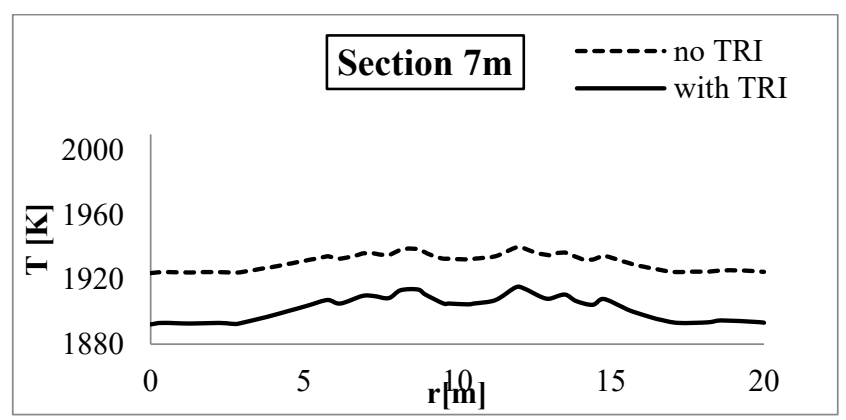

(a)

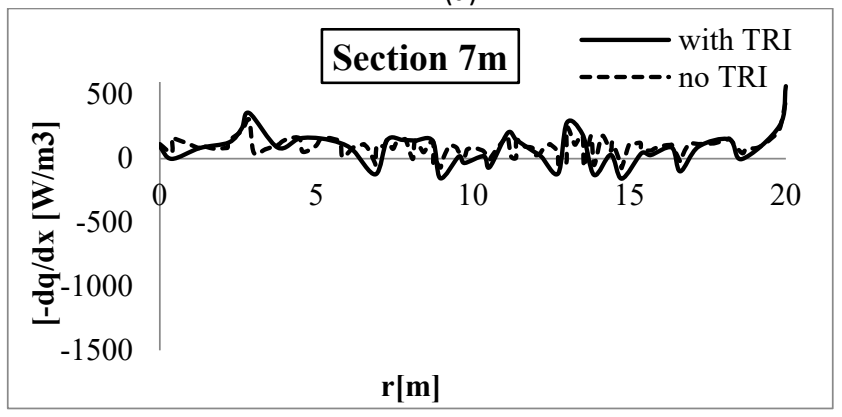

(b)

Fig. 4 (a) Temperature profile at section 7m, (b) Divergence of the radiative flux at section $7 \mathrm{~m}$. 


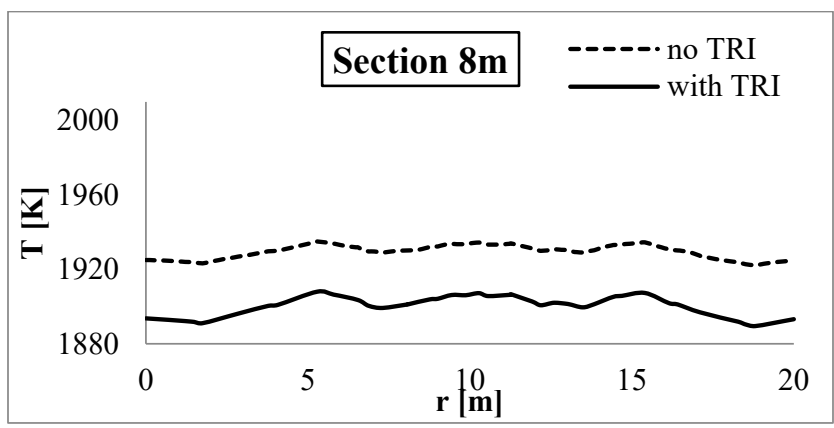

(a)

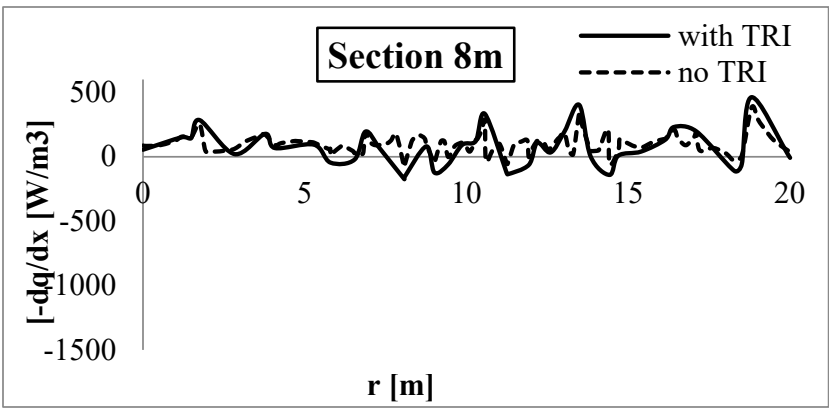

(b)

Fig. 5 (a) Temperature profile at section $8 \mathrm{~m}$, (b) Divergence of the radiative flux at section $8 \mathrm{~m}$.

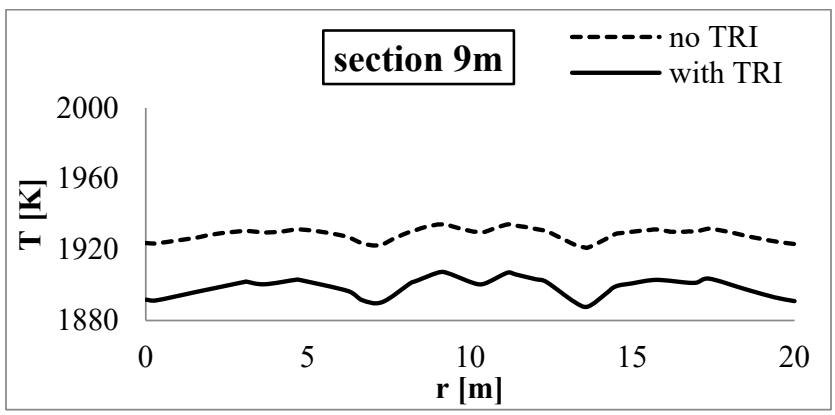

(a)

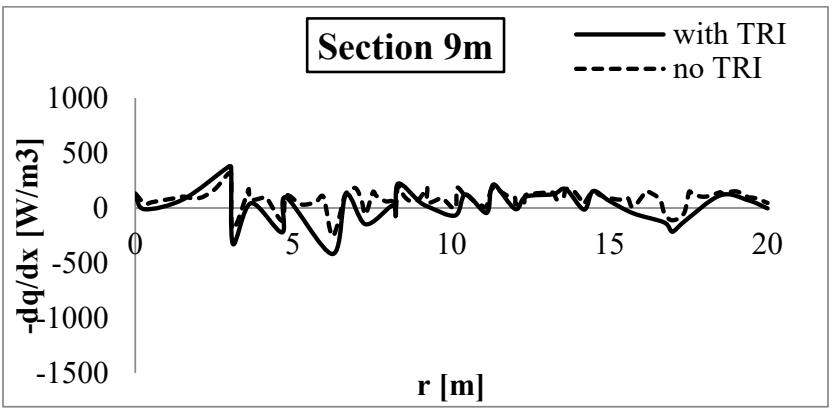

(b)

Fig. 6 (a) Temperature profile at section 9m, (b) Divergence of the radiative flux at section $9 \mathrm{~m}$

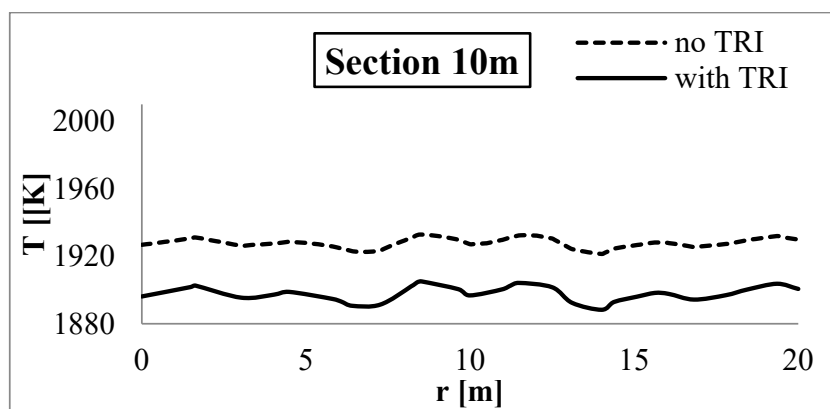

(a)

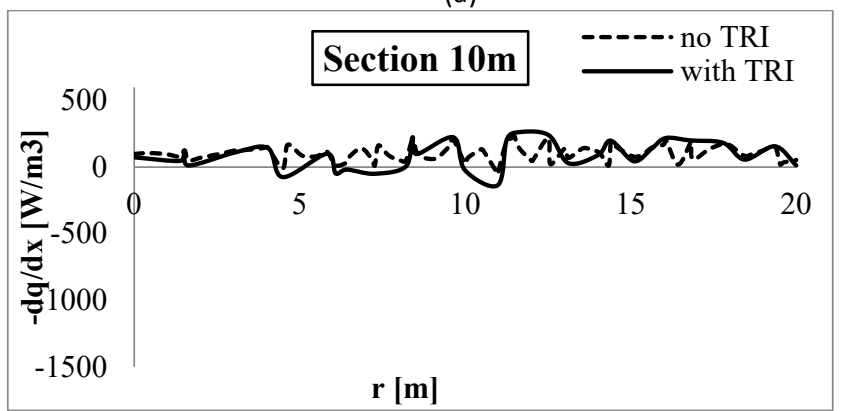

(b)

Fig. 7 (a) Temperature profile at section 10m, (b) Divergence of the radiative flux at section $10 \mathrm{~m}$.

The magnitude of the fluctuating axial velocity across the chamber is shown to decrease significantly as shown in Fig. (8). From the upward half of the chamber, the axial velocity becomes fairly constant as the influence of the turbulence flow field degenerates due to spectral distributions of the turbulent kinetic energy which is at its maximum at the inlet, as shown in Fig. (9), then, gradually retarding uniformly towards the outlet of the chamber.

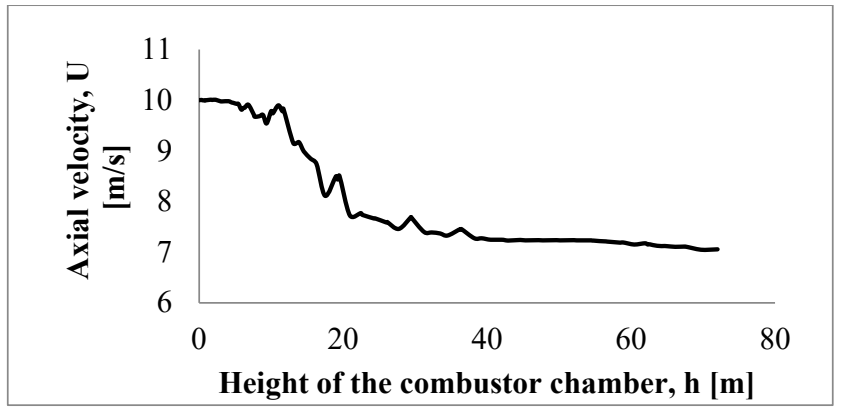

Fig. 8 Magnitude of the axial centerline velocity.

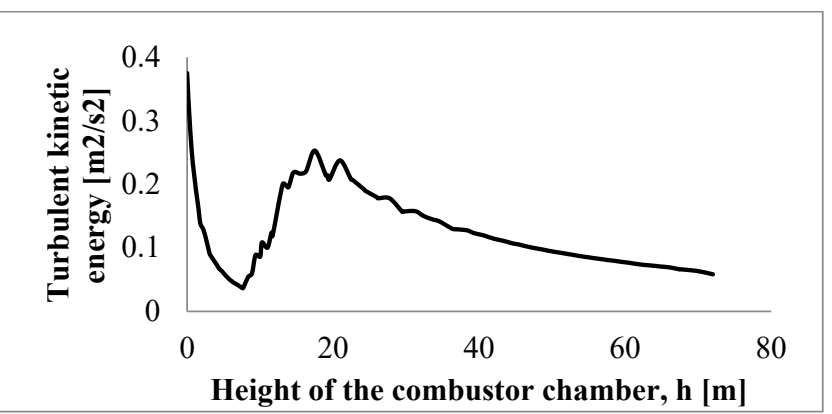

Fig. 9 Turbulent kinetic energy of the combustor. 
Analysis of the result at the critical section of the combustor $(6 \mathrm{~m})$ in order to investigate the critical factor for the deviation of the mean divergence of the radiative flux taking into consideration the effect of turbulence in the thermal radiation field is presented in Table 3. It can be inferred that turbulence-radiation interactions causes radiative heat losses from the flame, with the divergence of the radiative heat flux having a deviation factor of 3.48 , and a $1.43 \%$ reduction in the local flame temperature.

Table 3 Mean divergence of the radiative flux at section $6 \mathrm{~m}$.

\begin{tabular}{|l|c|c|c|c|}
\hline \multicolumn{1}{|c|}{ Cases } & $\begin{array}{c}\text { Mean } \\
\text { Temperature } \\
{[K]}\end{array}$ & $\begin{array}{c}\text { Temperature } \\
\text { difference } \\
{[\mathrm{K}]}\end{array}$ & $\begin{array}{c}\text { Divergence } \\
\text { of the heat } \\
\text { flux }\left[\mathrm{KW} / \mathrm{m}^{3}\right]\end{array}$ & Deviation \\
\hline $\begin{array}{l}\text { Isolated } \\
\text { radiation } \\
\text { field }\end{array}$ & 1932.81 & - & 50.29 & - \\
\hline $\begin{array}{l}\text { Radiation } \\
\text { coupled with } \\
\text { turbulence } \\
\text { flow }\end{array}$ & 1905.23 & $\begin{array}{c}-27.58 \\
(1.43 \%)\end{array}$ & 225.48 & +3.48 \\
\hline
\end{tabular}

\section{CONCLUSION}

A numerical simulation of the effect of turbulence flow field in the thermal-radiation field has been studied using COSMOL Mutiphysics software by analyzing and comparing five different sections of the combustor chamber. The results showed that Turbulence-Radiation Interactions (TRI) increase the heat loss from the gas turbine combustor flame, leading to a reduction in the local flame temperature as observed from their profiles. Consequently, the radiation heat source term which is synonymous with the divergence of the radiative heat flux is increased. This term is an integral part of the total energy equation, inserted as a source term; though, often neglected in low temperature processes.

Further, the magnitude of the fluctuating axial velocity across the combustor chamber decreases significantly, as the influence of the turbulence flow field degenerates due to spectral distributions of the turbulent kinetic energy.

Hence, the importance of taking into consideration the influence of turbulence in the thermal radiation field generated by the high energy, combustion process in a gas turbine combustor cannot be overemphasized. Analysis of the critical section of the combustor chamber resulted in deviation of the divergence of the radiative heat flux up to a factor of 3.48 , with $1.43 \%$ reduction in the local flame temperature.

\section{REFERENCES}

Barve, V. V., Clemens, N. T., Ezekoye, O. A., and Katta, V. R., 2006, "Numerical Study of the Evolution of Strongly Forced Axisymmetric Laminar Cold-Flow Jets," AIAA Journal, 44(8), 1742-1752.

https://doi.org/10.2514/1.17983

Coelho, P. J., 2007, "Numerical simulation of the interaction between turbulence and radiation in reactive flows," Progr. Energy Combust. Sci., 33(4), 311-383.

https://doi.org/10.1016/j.pecs.2006.11.002

Dos Santos, E. D., Galarca, M. M., Mossi, A. C., Petry, A. P., and Franca, F. H. R., 2009, "A Numerical Study of the Influence of Temperature Fluctuations in the Thermal Radiation Field," Thermal Engineering, 8, 51-57.

https://doi.org/10.1109/MCSUL.2009.12
Faeth, G. M., Gore, J. P., Chuech, S. G, and Jeng, S.M., 1989, "Radiation from turbulent diffusion flames," In Annual Review of Numerical Fluid Mechanics and Heat Transfer, 2, 1-38, Hemisphere, Washington, D.C. https://doi.org/10.1615/AnnualRevHeatTransfer.v2.30

Germano, M., 1978, "Turbulent fluctuations coupled with the radiation field," AIAA Paper, 78, 780-840.

https://doi.org/10.2514/6.1978-840

Li, G., and Modest, M. F., 2002, "Application of composition PDF methods in the investigation of turbulence-radiation interactions," Journal of Quantitative Spectroscopy \& Radiative Transfer, 73, 461-472. https://doi.org/10.1016/S0022-4073(01)00218-7

Li, G., and Modest, M. F., 2003, "Importance of turbulence-radiation interactions in turbulent diffusion flames," Journal of Heat Transfer, 125, 831-838.

$\underline{\text { https://doi.org/10.1115/1.1597621 }}$

Masataka Mochizuki, Koichi Mashiko, Thang Nguyen, 2019, "Data Center Energy Conservation by Heat Pipe Based Pre-Cooler System," Frontiers in Heat and Mass Transfer, 13, 24.

https://doi.org/10.5098/hmt.13.24

Ian M. Kennedy, 1997, "Models of soot formation and oxidation," Progress in Energy and Combustion Science, 23(2), 95-132. https://doi.org/10.1016/S0360-1285(97)00007-5

Modest, M. F., 2003, Radiative heat transfer, 2nd ed., Academic press, New York.

https://doi.org/10.1016/B978-012503163-9/50023-0

Babuska Ivo, and Oden Tinsley, J., 2004, "Verification and validation in computational engineering and science: basic concepts," Computer Methods in Applied Mechanical Engineering, Elsevier, 193, 4057-4066. https://doi.org/10.1016/j.cma.2004.03.002

Riyadh S. Al-Turaihi, Doaa Fadhil, Azher M. Abed, 2019, "Effect of Semi-circle Rib on Heat Transfer Coefficient in a Rectangular Channel," Frontiers in Heat and Mass Transfer, 13, 29.

https://doi.org/10.5098/hmt.13.29

Song, T. H., and Viskanta, R., 1987, "Interaction of turbulence with radiation: application to combustion systems," AIAA Journal of Thermophysics and Heat Transfer, 1, 56-62.

https://doi.org/10.2514/3.7

Tessé, L., Dupoirieux, F., and Taine, J., 2004, "Monte Carlo Modeling of Radiative Transfer in a Turbulent Sooty Flame," International Journal of Heat and Mass Transfer, 47, 555-572.

https://doi.org/10.1016/j.ijheatmasstransfer.2003.06.003

Townsend, A. A., 1958, "The effects of radiative transfer on turbulent flow of a stratified fluid," Journal of Fluid mechanics, 4, 361-375. https://doi.org/10.1017/S0022112058000501

Barths, H., Hasse, C., and Peters, N., 2000, "Computational fluid dynamics modelling of non-premixed combustion in direct injection diesel engines," International Journal of Engine Research, 1(3), 249267.

https://doi.org/10.1243/1468087001545164 